\title{
Relevance of Dental Health Capacity and Pulmonary Function Status in Adults
}

\author{
Kwui-Sook Song, Da-Young Ryu ${ }^{1}$, and In-Ja Kim ${ }^{\dagger}$ \\ Department of Preventive and Public Health Dentistry, Wonkwang University College of Dentistry, Iksan 54538, \\ ${ }^{1}$ Department of Dental Hygiene, Hyejeon College, Hongseong 32244, Korea
}

The purpose of this study was to confirm the relevance of dental health capacity and pulmonary function status. The subjects of this study were adults aged 40 years and older, who participated in pulmonary function and oral examinations. The data used for this study were raw data from the sixth Korea National Health and Nutrition Examination Survey (2013 2015). Statistical analysis was performed by analysis of complex sample survey data. The results were as follows: First, restrictive and obstructive patterns were more prevalent among males than females. They were even worse in older subjects. Approximately $67 \%$ of smokers had obstructive pattern. Second, there was a relevance between dental health capacity and pulmonary function status. The functioning teeth index, number of present teeth, and number of sound teeth (ST), which are indicators of dental health, declined with decreased pulmonary function. The number of missing teeth (MT) increased as the pulmonary function deteriorated $(p<0.05)$. Third, the relevance between dental health capacity and pulmonary function status after stratifying the life-long smoking experience was statistically significant in the tissue health (T-health) index and ST among non-smokers. The relevance between dental health capacity and pulmonary function status after stratifying the life-long smoking experience was statistically significant $(p<0.05)$ for the T-health index, ST, and MT among smokers. However, there was no relevance between dental health capacity and pulmonary function status after stratifying the life-long smoking experience and adjusting confounding variables. In conclusion, there was a relevance between dental health capacity and pulmonary function status after confounding variables were adjusted. However, there was no relevance between dental health capacity and pulmonary function status after stratifying the life-long smoking experience and adjusting confounding variables.

Key Words: Lung, Oral health, Respiratory function tests, Tooth

\section{Introduction}

Respiratory diseases are reported worldwide. Generally, asthma occurs more commonly during childhood, chronic obstructive pulmonary diseases (COPDs) such as emphysema and chronic bronchitis are prevalent in late adulthood, and infectious diseases such as tuberculosis may occur in all age groups ${ }^{1)}$. According to the 2015 causes of death statistics, respiratory disease such as pneumonia is included in the top 10 major causes of death, steadily climbing the list from the 10th place in 2004 to fourth place in 2015 following cancer, heart disease, and cardiovascular disease ${ }^{2}$.

Pulmonary function test (PFT) is one of the basic tests for the respiratory system, which accurately reflects the pathophysiology of respiratory diseases. It is useful for diagnosing respiratory diseases through quantitative and qualitative assessment of pulmonary function and can be also used for treatment and progress monitoring of respiratory diseases. PFT measures forced vital capacity (FVC) and forced expiratory volume in 1 second $\left(\mathrm{FEV}_{1}\right)$ to calculate forced expiratory ratio $\left(\mathrm{FEV}_{1} / \mathrm{FVC}\right)$, and the calculated value is compared with the normal value to diagnose restrictive and obstructive ventilatory impairments based on the extent of difference in values. Restrictive ventilatory impairment refers to decreased FVC, where a reduction in lung volume occurs due to damage to the lung itself or thoracic anomalies, and 
typical diseases that can cause manifestation of restrictive ventilatory impairment include pleural disease, interstitial lung disease, and pulmonary fibrosis. Obstructive ventilatory impairment refers to airflow limitation caused by obstruction of the airway, and typical diseases that can cause manifestation of obstructive ventilatory impairment include COPD, emphysema, and asthma ${ }^{3-5)}$.

Meanwhile, oral health is closely associated with systemic diseases and can have an impact on respiratory diseases as well. A review of foreign precedent studies on oral health and respiratory diseases shows that dental plaque in the oral cavity of chronic lung disease patients acts as a repository for bacteria that can cause pneumonia $^{6}$, while periodontal disease-related bacteria present in saliva can attach to airway mucosa and destroy the protective membrane on the lung surface and compromise pulmonary defense mechanisms, causing respiratory diseases ${ }^{7}$. Moreover, it has also been reported that dental plaque that causes oral diseases contains about 100 million bacteria per $1 \mathrm{~mm}^{2}$ of area and continuously supplies potential pathogens to the mouth and airways, which may enter the lungs through saliva and the upper respiratory tract ${ }^{8)}$. There are also claims that COPD can be prevented by improving periodontal status and oral hygiene ${ }^{9)}$. In Korea, a study analyzed data from the fourth Korea National Health and Nutrition Examination Survey (KNHANES) and reported that there was no association between periodontal disease and COPD ${ }^{10)}$, while another analyzed data from the 2012 KNHANES to investigate the association between the number of natural teeth and COPD and found that having fewer natural teeth was associated with higher probability of COPD, but such association appeared only in males ${ }^{11)}$. Another study investigated the association between the number of existing natural teeth and COPD using 2009 KNHANES data and found that having fewer numbers of existing natural teeth increased the risk of COPD ${ }^{12}$.

Thus far, studies in Korea and abroad examined the associations of lung disease with oral hygiene, number of natural teeth, and periodontal disease, but unfortunately, most of them have focused only on periodontal health and COPD. In light of this, the present study aimed to investigate the association between dental health and pulmonary function status using dental health indicators (tissue health [T-health] index, functioning teeth [FS-T] index, number of sound teeth [ST], number of present teeth [PT], and number of missing teeth [MT]) that can objectively identify oral health status.

\section{Materials and Methods}

\section{Subjects}

The present study used raw data from the sixth KNHANES (2013 2015) and secondary analysis after obtaining Institutional Review Board approval/exemption from Wonkwang University (IRB no. WKIRB-201707SB-042). The present study included 6,170 subjects, aged 40 years or older, who had participated in the health questionnaire survey, oral examination, and PFT.

\section{Methods}

In the present study, demographic factors, pulmonary function status, and dental health were used as the variables. Demographic factors included gender, age, income level, education level, lifetime drinking experience, lifetime smoking experience, and obesity status. Income level was divided into income quartiles: "upper," "upper-middle," "lower-middle," and "lower." Education level was divided into "primary school or below," "middle school," "high school," and "college or above." Lifetime drinking experience was divided into "no experience of drinking" and "yes," while lifetime smoking experience was also divided into "no experience of smoking" and "yes." Meanwhile, obesity was divided into "underweight," "normal," and "obese."

Pulmonary function status was based on PFT results and divided into "normal pulmonary function," "restrictive ventilatory impairment," and "obstructive ventilatory impairment." "Normal pulmonary function" was defined as $\mathrm{FEV}_{1} / \mathrm{FVC} \geq 0.7$ and FVC $\geq 0.8$, "restrictive ventilatory impairment" was defined as normal $\mathrm{FEV}_{1} / \mathrm{FVC}$ and FVC $<0.8$, and "obstructive ventilatory impairment" was defined as $\mathrm{FEV}_{1} / \mathrm{FVC}<0.7^{3,12)}$.

Dental health-related variables were derived from 28 permanent teeth, excluding the third molars. Dental health-related indicators included T-health index, FS-T 
index, PT, ST, and MT. In the T-health index, 4 points were given for ST, 2 points for filled teeth, 1 point for teeth with caries, and 0 point for MT. The total score ranged between 0 and 112 points with different weighted values applied according to the condition of the tooth. FS-T index represented the sum value of sound and filled teeth scores added, and the total score ranged between 0 and 28 points, under the concept that sound and filled teeth have the same function ${ }^{13}$.

\section{Analytic methods}

The present study used raw data from the sixth KNHANES (2013 2015), and complex sample analysis was performed to ensure that the analysis results were highly representative. Before performing complex sample analysis, kstrata, primary sampling unit, and weight values for healthy questionnaire and examination were applied to generate a plan file. For investigation of pulmonary function status based on demographic characteristics, complex sample cross-analysis and complex sample general linear model analysis were performed; however, for investigation of dental health based on pulmonary function status, complex sample general linear model analysis was performed. For an in-depth investigation of the association between pulmonary function status and dental health, complex sample multiple logistic regression analysis was performed, and smoking experience, a major risk factor for pulmonary function impairment, was stratified for analysis of the association between pulmonary function status and dental health. IBM SPSS Statistics ver. 23.0 (IBM Co., Armonk, NY, USA) was used for statistical analysis, and type I error level $(\alpha)$ for determining statistical significance was set to 0.05 .

Table 1. Pulmonary Function Status according to Demographic Distribution

\begin{tabular}{|c|c|c|c|c|}
\hline \multirow{2}{*}{ Classification } & \multicolumn{3}{|c|}{ Pulmonary function status } & \multirow{2}{*}{ p-value } \\
\hline & Normal $(n=5,005)$ & Restrictive pattern $(n=499)$ & Obstructive pattern $(n=666)$ & \\
\hline \multicolumn{5}{|l|}{ Gender } \\
\hline Male & $1,881(44.9)$ & $240(54.1)$ & $488(76.4)$ & \multirow[t]{2}{*}{$<0.001^{\mathrm{a}}$} \\
\hline Female & $3,124(55.1)$ & 259 (45.9) & $178(23.6)$ & \\
\hline Age (y) & $51.58 \pm 0.15$ & $55.37 \pm 0.48$ & $59.63 \pm 0.48$ & $<0.001^{\mathrm{b}}$ \\
\hline \multicolumn{5}{|l|}{ Income } \\
\hline Low & $1,073(22.1)$ & $112(23.1)$ & $147(23.9)$ & \multirow[t]{4}{*}{0.426} \\
\hline Low-middle & $1,240(25.0)$ & $108(23.4)$ & $155(22.5)$ & \\
\hline High-middle & $1,273(25.1)$ & $131(27.9)$ & $182(28.6)$ & \\
\hline High & $1,392(27.8)$ & $146(25.6)$ & $177(25.1)$ & \\
\hline \multicolumn{5}{|l|}{ Education } \\
\hline$\leq$ Primary school & $879(14.4)$ & $127(22.5)$ & $210(29.1)$ & \multirow[t]{4}{*}{$<0.001^{\mathrm{a}}$} \\
\hline Middle school & $647(12.7)$ & $73(13.0)$ & $105(16.2)$ & \\
\hline High school & $1,772(39.9)$ & $155(34.5)$ & $194(32.3)$ & \\
\hline$\geq$ College & $1,465(33.0)$ & $124(30.0)$ & $133(22.4)$ & \\
\hline \multicolumn{5}{|l|}{ Drinking experience } \\
\hline No & $577(9.8)$ & $76(12.3)$ & $64(8.9)$ & \multirow[t]{2}{*}{0.209} \\
\hline Yes & $4,286(90.2)$ & $405(87.7)$ & $581(91.1)$ & \\
\hline \multicolumn{5}{|l|}{ Smoking experience } \\
\hline No & 3,167 (59.6) & $291(53.5)$ & $233(33.5)$ & \multirow[t]{2}{*}{$<0.001^{\mathrm{a}}$} \\
\hline Yes & $1,694(40.4)$ & $188(46.5)$ & $412(66.5)$ & \\
\hline \multicolumn{5}{|l|}{ Body weight } \\
\hline Under weight & $92(1.8)$ & $12(1.9)$ & $9(1.4)$ & \multirow[t]{3}{*}{$<0.001^{\mathrm{a}}$} \\
\hline Nomal & $3,233(64.1)$ & 215 (41.4) & 439 (64.3) & \\
\hline Obesity & $1,680(34.0)$ & $271(56.7)$ & $218(34.3)$ & \\
\hline
\end{tabular}

Values are presented as $\mathrm{n}(\%)$ or mean \pm standard error.

${ }^{a} \mathrm{p}<0.05$, $\mathrm{p}$-value obtained from complex sample chi-square test. ${ }^{\mathrm{b}} \mathrm{p}<0.05$, $\mathrm{p}$-value obtained from complex sample general linear model. 


\section{Results}

\section{Pulmonary function status based on demographic characteristics}

Pulmonary function status based on demographic characteristics is shown in Table 1. Among the demographic characteristics, statistically significant differences in pulmonary function status were based on gender, age, education level, lifetime smoking experience, and obesity ( $\mathrm{p}<0.05)$. For pulmonary function status based on gender, there were more females $(55.1 \%)$ than males (44.9\%) in the normal pulmonary function group, approximately 1.2 times more males $(54.1 \%)$ than females (45.9\%) in the restrictive ventilatory impairment group, and approximately three times more males (76.4\%) than females (23.6\%) in the obstructive ventilatory impairment group. For pulmonary function status based on age, the obstructive ventilatory impairment group had the highest average age (59.63 years), while the normal pulmonary function group had the lowest average age (51.58 years). With respect to pulmonary function status based on education level, high school was most common for all three groups: normal pulmonary function (39.9\%), restrictive ventilatory impairment (34.5\%), and obstructive ventilatory impairment $(32.3 \%)$. There were more people with no lifetime smoking experience in the normal pulmonary function and restrictive ventilatory impairment groups (59.6\% and 53.5\%, respectively), while there were more people with smoking experience in the obstructive ventilatory impairment group (66.5\%). There were more people with normal weight in the normal pulmonary function and obstructive ventilatory impairment groups
(64.1\% and $64.3 \%$, respectively), while there were more obese people in the restrictive ventilatory impairment group $(56.7 \%)$.

\section{Dental health based on pulmonary function status}

With respect to dental health based on pulmonary function status, significant differences were found in all categories, except T-health index $(\mathrm{p}<0.05)$. FS-T index showed the highest number of teeth with 25.25 in the normal pulmonary function group, followed by 24.31 in the restrictive ventilatory impairment group and 23.69 in the obstructive ventilatory impairment group, indicating that poorer pulmonary function status was associated with lower FS-T index. PT and ST were also the highest in the normal pulmonary function group (25.32 and 23.61, respectively) and the lowest in the obstructive ventilatory impairment group (23.77 and 21.49, respectively). MT in the normal pulmonary function, restrictive ventilatory impairment, and obstructive ventilatory impairment groups was $0.97,1.13$, and 1.49 , respectively, indicating that poorer pulmonary function status was associated with higher MT (Table 2).

\section{Association between dental health and pulmonary function status}

Table 3 shows the results from multiple logistic regression analysis on the association between dental health and pulmonary function status and on relevant factors. After adjusting for gender, age, income level, education level, lifetime drinking experience, lifetime smoking experience, and obesity, the results showed statistically significant differences in ST and MT (p

Table 2. Pulmonary Function Status according to Dental Health Capacity

\begin{tabular}{lrcrrr}
\hline \multirow{2}{*}{ Classification } & \multicolumn{4}{c}{ Pulmonary function status $^{*}$ p-value $^{2}$} \\
\cline { 2 - 4 } & Normal $^{\mathrm{a}}$ & Restrictive pattern $^{\mathrm{b}}$ & Obstructive pattern $^{\mathrm{c}}$ & 0.107 \\
T-health & $98.91 \pm 0.18$ & $98.55 \pm 0.49$ & $97.81 \pm 0.51$ & $<0.001$ \\
FS-T & $25.25 \pm 0.06$ & $24.31 \pm 0.22^{\mathrm{a}>\mathrm{b}}$ & $23.69 \pm 0.20^{\mathrm{a}>\mathrm{c}}$ & $<0.001$ \\
PT & $25.32 \pm 0.06$ & $24.40 \pm 0.22^{\mathrm{a}>\mathrm{b}}$ & $23.77 \pm 0.19^{\mathrm{a}>\mathrm{c}}$ & $<0.001$ \\
ST & $23.61 \pm 0.08$ & $22.55 \pm 0.25^{\mathrm{a}>\mathrm{b}}$ & $21.49 \pm 0.24^{\mathrm{a}>\mathrm{c}}$ & $<0.001$ \\
MT & $0.97 \pm 0.03$ & $1.13 \pm 0.08$ & $1.49 \pm 0.08^{\mathrm{a}<\mathrm{c}}$ & \\
\hline
\end{tabular}

Values are presented as mean \pm standard error.

T-health: tissue health, FS-T: functioning teeth, PT: present teeth, ST: sound teeth, MT: missing teeth.

$\mathrm{p}<0.05$, $\mathrm{p}$-value obtained from complex sample general linear model. 
Table 3. Relationship between Dental Health Capacity and Pulmonary Function Status

\begin{tabular}{|c|c|c|c|c|c|c|}
\hline \multirow{2}{*}{ Classification } & \multirow{2}{*}{$\begin{array}{c}\text { Restrictive pattern } \\
\text { OR }(95 \% \text { CI })\end{array}$} & \multirow{2}{*}{$\begin{array}{c}\text { Obstructive pattern } \\
\text { OR }(95 \% \mathrm{CI})\end{array}$} & \multirow{2}{*}{$\mathrm{p}$-value } & \multirow{2}{*}{$\begin{array}{c}\text { Restrictive pattern } \\
\text { Adjust OR }(95 \% \mathrm{CI})^{\mathrm{a}}\end{array}$} & \multirow{2}{*}{$\begin{array}{c}\text { Obstructive pattern } \\
\text { Adjust OR }(95 \% \mathrm{CI})^{\mathrm{a}}\end{array}$} & \multirow{2}{*}{$\mathrm{p}$-value } \\
\hline & & & & & & \\
\hline T-health & $1.01(0.99 \sim 1.03)$ & $1.07(1.04 \sim 1.10)$ & $<0.001$ & $1.00(0.97 \sim 1.02)$ & $1.03(1.00 \sim 1.06)$ & 0.119 \\
\hline FS-T & $0.88(0.69 \sim 1.13)$ & $0.98(0.78 \sim 1.23)$ & 0.621 & $0.92(0.71 \sim 1.20)$ & $1.29(0.95 \sim 1.75)$ & 0.193 \\
\hline PT & $1.10(0.86 \sim 1.42)$ & $1.19(0.93 \sim 1.53)$ & 0.320 & $1.03(0.79 \sim 1.35)$ & $0.87(0.63 \sim 1.20)$ & 0.646 \\
\hline ST & $0.96(0.90 \sim 1.02)$ & $0.80(0.75 \sim 0.86)$ & $<0.001$ & $0.93(0.92 \sim 1.05)$ & $0.85(0.79 \sim 0.90)$ & $<0.001$ \\
\hline MT & $0.98(0.88 \sim 1.10)$ & $1.32(1.17 \sim 1.49)$ & $<0.001$ & $0.91(0.81 \sim 1.03)$ & $1.12(0.99 \sim 1.26)$ & 0.048 \\
\hline
\end{tabular}

OR: odds ratio, $95 \%$ CI: 95\% confidence interval, T-health: tissue health, FS-T: functioning teeth, PT: present teeth, ST: sound teeth, MT: missing teeth.

$\mathrm{p}<0.05$, $\mathrm{p}$-value obtained from complex sample multinomial logistic regression.

${ }^{\mathrm{a}}$ Gender, age, income, education, drinking experience, smoking experience, body weight.

Table 4. Pulmonary Function Status in Relation to Dental Health Capacity and Stratification of Smoking Experience

\begin{tabular}{|c|c|c|c|c|c|c|}
\hline \multirow{2}{*}{ Classification } & \multirow{2}{*}{$\begin{array}{c}\text { Restrictive pattern } \\
\text { OR }(95 \% \mathrm{CI})\end{array}$} & \multirow{2}{*}{$\begin{array}{c}\text { Obstructive pattern } \\
\text { OR }(95 \% \mathrm{CI})\end{array}$} & \multirow{2}{*}{$\mathrm{p}$-value } & \multirow{2}{*}{$\begin{array}{c}\text { Restrictive pattern } \\
\text { Adjust OR }(95 \% \mathrm{CI})^{\mathrm{a}}\end{array}$} & \multirow{2}{*}{$\begin{array}{c}\text { Obstructive pattern } \\
\text { Adjust OR }(95 \% \mathrm{CI})^{\mathrm{a}}\end{array}$} & \multirow{2}{*}{ p-value } \\
\hline & & & & & & \\
\hline \multicolumn{7}{|l|}{ Non-smoker } \\
\hline T-health & $1.01(0.98 \sim 1.04)$ & $1.03(1.00 \sim 1.05)$ & 0.040 & $0.99(0.96 \sim 1.02)$ & $1.00(0.96 \sim 1.04)$ & 0.634 \\
\hline FS-T & $0.83(0.61 \sim 1.12)$ & $1.03(0.62 \sim 1.70)$ & 0.457 & $0.86(0.62 \sim 1.21)$ & $1.06(0.59 \sim 1.89)$ & 0.641 \\
\hline PT & $1.15(0.84 \sim 1.56)$ & $1.05(0.63 \sim 1.74)$ & 0.620 & $1.05(0.75 \sim 1.48)$ & $0.97(0.53 \sim 1.77)$ & 0.951 \\
\hline ST & $0.97(0.90 \sim 1.05)$ & $0.84(0.79 \sim 0.89)$ & $<0.001$ & $1.04(0.95 \sim 1.13)$ & $0.93(0.85 \sim 1.02)$ & 0.187 \\
\hline MT & $0.97(0.84 \sim 1.12)$ & $1.19(1.00 \sim 1.42)$ & 0.125 & $0.88(0.75 \sim 1.02)$ & $0.93(0.78 \sim 1.11)$ & 0.173 \\
\hline \multicolumn{7}{|l|}{ Smoker } \\
\hline T-health & $1.01(0.97 \sim 1.05)$ & $1.06(1.02 \sim 1.09)$ & 0.013 & $0.99(0.95 \sim 1.03)$ & $1.01(0.98 \sim 1.05)$ & 0.544 \\
\hline FS-T & $0.95(0.64 \sim 1.41)$ & $1.13(0.84 \sim 1.51)$ & 0.661 & $0.99(0.65 \sim 1.50)$ & $1.38(0.96 \sim 1.97)$ & 0.198 \\
\hline PT & $1.05(0.69 \sim 1.59)$ & $1.03(0.75 \sim 1.41)$ & 0.966 & $0.97(0.63 \sim 1.50)$ & $0.77(0.53 \sim 1.12)$ & 0.381 \\
\hline ST & $0.95(0.84 \sim 1.06)$ & $0.82(0.74 \sim 0.89)$ & $<0.001$ & $1.02(0.90 \sim 1.16)$ & $0.94(0.86 \sim 1.04)$ & 0.405 \\
\hline MT & $0.97(0.81 \sim 1.15)$ & $1.29(1.10 \sim 1.51)$ & 0.006 & $0.88(0.73 \sim 1.06)$ & $1.08(0.93 \sim 1.26)$ & 0.176 \\
\hline
\end{tabular}

OR: odds ratio, 95\% CI: 95\% confidence interval, T-health: tissue health, FS-T: functioning teeth, PT: present teeth, ST: sound teeth, MT: missing teeth.

$\mathrm{p}<0.05$, $\mathrm{p}$-value obtained from complex sample multinomial logistic regression.

${ }^{a}$ Gender, age, income, education, drinking experience, body weight.

$<0.05)$. Compared with ST of people with normal pulmonary function status, higher ST was associated with higher odds ratio of having restrictive ventilatory impairment by 0.93 times and of having obstructive ventilatory impairment by 0.85 times. Compared with MT of people with normal pulmonary function status, higher MT was associated with higher odds ratio of having restrictive ventilatory impairment by 0.91 times and of having obstructive ventilatory impairment by 1.12 times.

\section{Association between dental health and pulmonary function status based on lifetime smoking experi- ence of the subject}

Table 4 shows the results from analyzing the association between dental health and pulmonary function status and relevant factors, with lifetime smoking experience being stratified. After stratifying lifetime smoking experience, the association between dental health and pulmonary function status showed statistically significant T-health index and ST among non-smokers and statistically significant T-health index, ST, and MT among smokers ( $\mathrm{p}$ $<0.05)$. However, when gender, age, income level, education level, lifetime drinking experience, lifetime smoking experience, and obesity were adjusted after stratifying lifetime smoking experience, smokers and non-smokers showed no statistically significant difference in the association between dental health and pulmonary function status $(p>0.05)$. 


\section{Discussion}

The oral cavity acts as a repository for respiratory pathogens to form colonies in the oropharynx, and when respiratory pathogens form colonies due to poor oral hygiene or periodontal disease, it may lead to respiratory diseases such as pneumonia ${ }^{14)}$. Especially in cases involving immunosuppressed patients, chronic infection inside the oral cavity has been identified to be associated with onset of respiratory diseases ${ }^{8}$. Determination of respiratory diseases is made by $\mathrm{PFT}^{3)}$, and since it was believed that restrictive and obstructive ventilatory impairments determined by PFT may be associated with oral health, the present study investigated the association between pulmonary function status and dental health, which can objectively represent oral health status.

In the present study, there were about 1.2 times as many males than females with restrictive ventilatory impairment and about 3 times as many males than females with obstructive ventilatory impairment. Pulmonary function status was worse as age increased, obstructive ventilatory impairment was more prevalent among smokers, and restrictive ventilatory impairment was more prevalent among those who were obese. Wang et al. ${ }^{15)}$ divided adults 30 years and older into the COPD $(n=306)$ and control $(n=328)$ groups for comparison and found that the average age of the COPD group was higher than that of the control group and the COPD group had about 2.2 times as many males than females and more current smokers. Deo et al. ${ }^{16)}$ recruited subjects 20 years and older and compared the COPD $(n=150)$ and control $(n=50)$ groups; they found that the COPD group had about 14 times as many males than females, had about two times as many people with low income than those with high income, and had about 11 times as many smokers than non-smokers. Shin et al. ${ }^{12)}$ analyzed 2009 KNHANES data of adults 40 years and older and found that there were about three times as many males than females among COPD patients and that more COPD patients tended to be 65 years or older, had low education level, and were smokers. However, since gender, age, body weight, and smoking status ${ }^{17,18)}$ are risk factors known to affect pulmonary function, caution should be taken when interpreting such results.
The present study identified that having poor pulmonary function status was associated with poor dental health. FS-T index is based on the presumption that ST and treated teeth may function in the same manner; in this study, the total number of these two types of teeth was added, and thus, a higher score would indicate healthier teeth. Poorer pulmonary function status resulted in lower FS-T index as well as fewer PT and ST. Wang et al. ${ }^{15)}$ reported that the COPD group had fewer natural teeth than the control group, while Shin et al. ${ }^{12)}$ reported that having fewer existing natural teeth was associated with higher risk of developing COPD. In a study by Kim et al. ${ }^{11)}$ who analyzed 2012 KNHANES data from subjects 40 years and older, the number of natural teeth in normal pulmonary function, restrictive ventilatory impairment, and obstructive ventilatory impairment groups was 25.4, 24.6, and 21.8 among males and 24.4, 23.7, and 21.4 among females, respectively. The results showed that having fewer natural teeth was associated with poorer pulmonary function status, which was consistent with the findings in the present study. On the other hand, MT increased with poorer pulmonary function status. A cohort study by Barros et al. ${ }^{19)}$ reported that incidence of COPD was approximately 2.3 times higher in edentulous patients; moreover, a study by Shin et al. ${ }^{12)}$ reported that people who wear dentures had higher probability of COPD and that those who wear a full denture had even higher probability of COPD than those who wear a partial denture, which supported the findings in the present study.

The present study confirmed that after adjusting for gender, age, income level, education level, lifetime drinking experience, lifetime smoking experience, and obesity as confounding variables, ST and MT were associated with pulmonary function status. Smoking is known as a major risk factor that affects pulmonary function ${ }^{17,18,20)}$, and since it acts as an effect modifier and a confounding factor in the relationship between periodontal disease and $\mathrm{COPD}^{21)}$, it was determined that smoking status should be taken into account when analyzing the association between dental health and pulmonary function status. Accordingly, the present study investigated the association between dental health and pulmonary function status after stratifying smoking experience and found that 
T-health index and ST were associated with pulmonary function status among non-smokers, while T-health index, ST, and MT were associated with pulmonary function status among smokers. In a study by Liu et al. ${ }^{9)}$ that investigated exacerbation of COPD, oral hygiene, and periodontal health in subjects 30 years or older, exacerbation of COPD was greater when there were fewer natural teeth, plaque index was higher, and tooth brushing frequency was lower among non-smokers and former smokers. However, when smoking experience was stratified and confounding variables were adjusted, the present study did not find statistically significant differences in dental health and pulmonary function status between smokers and non-smokers. Wang et al. ${ }^{15}$, who studied periodontal health, oral health behavior, and COPD in subjects 30 years and older, reported that after stratifying smoking status and adjusting for age, gender, and BMI index, fewer natural teeth had 1.05 times higher probability of COPD among non-smokers and higher dental plaque index had 2.34 times higher probability of COPD among current smokers. Summarizing the results from the present study and other precedent studies, smoking experience was associated with dental health and pulmonary function status. However, the association between dental health and pulmonary function status was not observed when smoking experience was stratified and confounding variables were adjusted. It is believed that this is due to the fact that since the present study was a cross-sectional study, it may have had limitations in identifying the mediating effects of smoking experience. Moreover, since other studies are showing different results on the mediating effects of smoking, the mediating effects of smoking experience could not be accurately determined. Therefore, it is believed that future longitudinal studies would be needed to confirm this.

Limitations in the present study included the fact that because precedent studies on T-health index, FS-T index, and pulmonary function status are so rare that the findings in the present study could not be compared and precedent studies on the association between other dental health indicators and pulmonary function status are also lacking, direct comparison with the present study was difficult. Moreover, because the present study was a cross-sectional study, it was difficult to identify the mediating effects of smoking experience in dental health and pulmonary function status, and thus, future longitudinal studies would be needed to identify the medicating effects of smoking experience in dental health and pulmonary function status. However, the present study was meaningful in that it was the first study that attempted to use reliable and representative raw data from the sixth KNHANES in investigating the association between pulmonary function status and dental health, which is an objective oral health indicator.

\section{References}

1. Nam BW: Neuropsychiatric aspects of pulmonary disease. Korean J Psychosom Med 17: 45-51, 2009.

2. Statistics Korea: National death cause statistics. Statistics Korea, Daejeon, 2015.

3. Korean Academy of Tuberculosis and Respiratory Diseases: Guideline for pulmonary function test in 2016. Medrang, Seoul, pp.3-33, 2016.

4. Korean Occupational Safety and Health Agency. Retrieved June 28, 2017, from http://oshri.kosha.or.kr/board?tc=Retrieve BoardViewCmd \&boardType=A\&contentId=364216\&pageNum= 1\&urlCode=T1|Y|404|371|371|404|||/board|N\&tabId=404/36 4216_20160329b.pdf(2016).

5. Kim YR, Lee SJ, Kim HJ, Lee SB: The significance of posture on assessment of pulmonary function after pulmonary rehabilitation in tetraplegia. J Korean Acad Rehabil Med 27: 513-518, 2003.

6. Didilescu AC, Skaug N, Marica C, Didilescu C: Respiratory pathogens in dental plaque of hospitalized patients with chronic lung diseases. Clin Oral Investig 9: 141-147, 2005.

7. Scannapieco FA: Role of oral bacteria in respiratory infection. J Periodontol 70: 793-802, 1999.

8. Paju S, Scannapieco FA: Oral biofilms, periodontitis, and pulmonary infections. Oral Dis 13: 508-512, 2007.

9. Liu Z, Zhang W, Zhang J, et al.: Oral hygiene, periodontal health and chronic obstructive pulmonary disease exacerbations. J Clin Periodontol 39: 45-52, 2012.

10. Jin HJ, Kim EK, An SY, Im SU, Song KB, Choi YH: Relationship between periodontal status and chronic obstructive pulmonary disease. J Korean Acad Oral Health 37: 147-153, 
2013.

11. Kim SW, Han K, Kim SY, Park CK, Rhee CK, Yoon HK: The relationship between the number of natural teeth and airflow obstruction: a cross-sectional study using data from the Korean National Health and Nutrition Examination Survey. Int J Chron Obstruct Pulmon Dis 11: 13-21, 2016.

12. Shin HS, Ahn YS, Lim DS: Association between the number of existing permanent teeth and chronic obstructive pulmonary disease. J Dent Hyg Sci 16: 217-224, 2016.

13. Sheiham A, Maizels J, Maizels A: New composite indicators of dental health. Community Dent Health 4: 407-414, 1987.

14. Jeong JS, Heo SM: The relationship between oral disease and respiratory disease in the elderly. J Korean Dent Assoc 51: 494-500, 2013.

15. Wang Z, Zhou X, Zhang J, et al.: Periodontal health, oral health behaviours, and chronic obstructive pulmonary disease. J Clin Periodontol 36: 750-755, 2009.

16. Deo V, Bhongade ML, Ansari S, Chavan RS: Periodontitis as a potential risk factor for chronic obstructive pulmonary disease: a retrospective study. Indian J Dent Res 20: 466-470, 2009.

17. Schoenberg JB, Beck GJ, Bouhuys A: Growth and decay of pulmonary function in healthy blacks and whites. Respir Physiol 33: 367-393, 1978.

18. Jenkins SC, Moxham J: The effects of mild obesity on lung function. Respir Med 85: 309-311, 1991.

19. Barros SP, Suruki R, Loewy ZG, Beck JD, Offenbacher S: A cohort study of the impact of tooth loss and periodontal disease on respiratory events among COPD subjects: modulatory role of systemic biomarkers of inflammation. PLoS One 8: e68592, 2013.

20. Hyman J: The importance of assessing confounding and effect modification in research involving periodontal disease and systemic diseases. J Clin Periodontol 33: 102-103, 2006.

21. Hyman JJ, Reid BC: Cigarette smoking, periodontal disease: and chronic obstructive pulmonary disease. J Periodontol 75: 9-15, 2004. 